\title{
Connectivity Investigation of Channel Quality-Based Adaptive Gossip Flooding Mechanism for AODV
}

\author{
Prasanna Shete ${ }^{1, *}$, Raval N Awale ${ }^{2}$ \\ ${ }^{1}$ Department of Computer Engineering, K. J. Somaiya College of Engineering, Mumbai, India \\ ${ }^{2}$ Veermata Jijabai Technological Institute, Mumbai, India \\ Received 28 February 2019; received in revised form 27 May 2019; accepted 15 August 2019
}

DOI: https://doi.org/10.46604/ijeti.2020.3812

\begin{abstract}
To address the "broadcast storm" problem associated with flooding-based route discovery mechanism of reactive routing protocols, probabilistic approaches are suggested in the literature. In the earlier work, Gossip flooding mechanism of Haas et.al. was extended with signal quality, to propose channel quality based adaptive gossip flooding mechanism for AODV (CQAG-AODV). Following the cross-layer design principle, CQAG-AODV algorithm tried to discover robust routes, as well as address the "broadcast storm" problem by controlling the rebroadcast probability of Route request (RREQ) packets on the basis of signal strength experienced at the physical layer. This paper investigates the connectivity of CQAG-AODV through theoretical and simulation analysis. Results show that, by accounting the signal strength in the route discovery process, not only does the proposed algorithm floods a lesser number of route requests and controls the broadcast storm, but also maintains a higher level of connectivity to offer high packet delivery ratio; independent of network density and node mobility. Moreover, due to controlled routing overhead and robust route discovery, channel quality based adaptive flooding mechanism offers fringe benefit of energy efficiency as well. CQAG-AODV thus proves its suitability in a variety of use cases of multi-hop ad hoc networks including WSNs and VANETs.
\end{abstract}

Keywords: multi-hop ad hoc networks, MANET, WSN, VANET, AODV, connectivity, cross-layer design

\section{Introduction}

Connectivity is the property of ad hoc networks that represents how well each node can reach every other node in the network via a multi-hop path. It is the fundamental pre-requisite for a network to be operational. Multi-hop ad hoc networks experience frequent path breaks mainly due to node or link failures; drastically degrading the system performance. For assuring Quality of Service (QoS), it is necessary that routing protocol should preserve the network connectivity. Link failures are the result of signal strength fluctuations, arising from time-varying characteristics of the wireless channel or node mobility. Thus in the routing protocol design, it is important to preserve link/path lifetime longer. This can be achieved by accounting the signal quality of links in the route discovery process.

The route discovery procedure of AODV counts on the flooding of special messages called Route requests (RREQs) [1]. The route to destination is discovered through flooding (i.e. multiple rebroadcasts) of RREQs by intermediate nodes. Flooding gives rise to two serious problems viz; "broadcast storm" [2-3], and resource consumption of nodes and network. Time-varying nature of wireless channel and node mobility are inherent characteristics of multi-hop ad hoc networks. Thus, if the discovered routes are contributed by weak links, they will experience frequent failures and hence efforts taken in discovering the routes 
will be wasted. Thus, blind flooding of RREQs without accounting the node or channel condition may result in wastage of node energy and network bandwidth.

In order to deal with the problem of "broadcast storm" in multi-hop ad hoc networks, various optimized flooding mechanisms are proposed [4-11]. These schemes advocate controlling the RREQ broadcasts adopting probabilistic approach, in which intermediate nodes forward the RREQs with lower probability based on some control metric such as node density, distance, number of neighbors covered, speed, etc. Reina et al. conducted an exhaustive survey of various probabilistic broadcast schemes proposed for optimizing the routing protocols of multi-hop wireless ad hoc networks [12].

Gossip based approach proposed in [4] uses fixed probability ( $p=0.66)$ for RREQ forwarding. It is shown that with Gossip $(1,0.66)$ almost $95 \%-99 \%$ of the time the network is connected, with almost $33 \%$ lesser number of RREQ being broadcast than simple flooding. A variation of gossip-based flooding, GOSSIP5 scheme is proposed in which the gossiping threshold is fixed to a number of node's neighbors [5]. Here, nodes wait for a fixed random delay before trying to retransmit the incoming packet. However, the proposed AODV+G and GOSSIP5 protocols did not comment about reducing link and/or path failures resulting from channel fluctuations. 'Neighbor Coverage-based Probabilistic Rebroadcast protocol (NCPR)' proposed in [6] utilizes neighbor coverage knowledge to determine how many neighbors should receive the RREQs. Based on the information about uncovered neighbors, connectivity metric and local node density, RREQ rebroadcast probability is calculated. Combining the neighbor coverage knowledge and probabilistic mechanism the number of redundant rebroadcast are significantly reduced. NCPR alleviates the network contention and collision thereby increasing packet delivery ratio and reducing the average end-to-end delay. The performance improvement of NCPR is significant in high density or heavy traffic networks, whereas in sparse networks performance is slightly better than flooding. However, if the uncovered nodes are not part of the path converging to the destination, route setup delay may be large. Zhang et al. suggested 'Estimated Distance (EstD)-based routing protocol EDRP' that limits the propagation range of RREQ messages [7]. EstD is an amalgamation of Estimated Geometrical Distance (EGD) and Estimated Topological Distance (ETD). EGD accounts the Received Signal Strength (RSS) variation at contact time of two nodes, to estimate the future geometrical distance between them when they move apart. Propagating RREQs in the direction of destination with the aid of EstD, considerably reduces the routing overhead and improves routing performance. However when node distribution is sparse, performance degrades significantly. Also, the use of gossiping, when a node does not have information about the destination, may adversely affect the performance. Hybrid flooding scheme that combines the features of probabilistic, neighbor based and area-based flooding is proposed in [8]. Here, based on neighborhood node density and the distance to neighbors, each node adjusts its forwarding probability. RREQs are flooded with low probability in the areas where node density is high, and with high probability otherwise. Also, forwarding of received RREQs is confined to the nodes located within a forwarding zone. Nodes outside the zone do not participate in RREQ forwarding. In dense networks, this scheme incurs less routing overhead as well as node energy consumption, as compared to simple flooding or static probabilistic flooding. In sparse networks, the performance is as good as with simple flooding. This scheme is beneficial only for nodes which are moving at speeds less than $36 \mathrm{~km} / \mathrm{hr}$; as speed increases routing load increases and thus makes it unsuitable in high mobility scenarios. Speed Adaptive Probabilistic Flooding (SAPF) algorithm proposed in [9] is an extension of probabilistic flooding, where the flooding probability is decided on the basis of node speed. Here the network density of VANETs is estimated based on the node's speed. The forwarding probability is adjusted by the following formula; $p=0.0557 v+0.033$, where $v$ is the speed of the vehicle. Two- speed thresholds are defined as $v_{l}$ and $v_{h}$. The authors indicated that if $v>v_{h}$, it is impossible to estimate the vehicle's density. On the other hand, if $v<v_{l}$, the network has almost reached its capacity and so the probability can be constant. A reliable and low-collision packet-forwarding scheme named Collision-Aware Reliable. Forwarding (CAREFOR) is proposed for VANETs [10]. In this, each vehicle receiving a packet rebroadcasts it with predefined probability. This probability is decided by the density of vehicles in the vicinity, distance between transmitting and receiving vehicles, and transmission range of the next-hop. However, the optimization approaches 
[5-10] are computationally intense and thus difficult to implement. Hence authors adopted a simple mechanism named 'Channel Quality based Adaptive Gossip Flooding mechanism for AODV (CQAG-AODV)' and presented performance improvement through the reduction in link failures [11]. By accounting signal quality in the routing process, CQAG-AODV tuned the rate of RREQ broadcasts and controlled the number of RREQs forwarded, as well as discovered good quality routes that are robust to failures.

Moreover, various optimization approaches were proposed to address the problems caused by interference [13-16]. In these approaches, Signal to Interference and Noise Ratio (SINR) experienced on the channel -called 'link status,' is utilized in the routing metrics design. However, these approaches hardly comment on the "broadcast storm" problem. Further, since link status information is loaded in the control packets (RREQ or Hello), these schemes will eventually increase routing overhead. Although, these approaches utilize the same metric of signal quality at the physical layer as suggested in [11], the proposed CQAG-AODV did not constitute interference in the routing decision. This is so because, in multi-hop wireless networks interference is less critical than connectivity [17-18]; interference mainly impacts the network capacity and not the connectivity. Thus, by selecting links/paths that offer improved signal strength will implicitly reduce the effect of interference. In this paper, we analyze the connectivity of CQAG-AODV. The main objective is to investigate whether CQAG-AODV discovers/selects best channel quality paths as well as addresses the "broadcast storm" problem while preserving the same level of connectivity as AODV.

The rest of paper is organized as follows; the channel quality based network model is presented in section 2 . Section 3 discusses channel quality based route discovery mechanism and implementation of proposed CQAG-AODV. Section 4 and 5 present theoretical and simulation-based performance analysis of CQAG-AODV. Section 6 concludes the paper.

\section{Channel Quality-based Network Model}

The received signal strength at a given point over a wireless medium is a function of its distance from the transmitter, represented as:

$$
P_{r}=\frac{P_{t} \cdot G_{t} \cdot G_{r}}{4 \pi d^{2}}
$$

where $P_{t}$ and $P_{r}$ are the transmitted and received signal power, $G_{t}$ and $G_{r}$ are gains of the transmitter and receiver antennas, and $d$ is a distance between transmitter and receiver.

Further, the received signal experiences variations due to multipath propagation effects, noise and interference. Thus received signal power keeps changing which can be represented by widely recognized time-varying multipath propagation model presented in [19], given as:

$$
y(t)=\sum_{i=1}^{p(t)} A_{i}(t) x\left(t-\tau_{i}(t)\right)+z(t)
$$

where $x(t)$ is the transmitted signal, $y(t)$ is the received signal, $z(t)$ is the background noise, $\tau_{i}(t)$ is the time delay, $p(t)$ is the number of paths and $A_{i}(t)$ is the attenuation of each path.

Moreover, node mobility affects the average channel coherence time. As per the Clarke and Gans model, the motion of nodes causes Doppler shift in the frequency of a received signal. The inverse of maximum Doppler frequency $\left(f_{m}\right)$ is known as the coherence interval $\left(T_{c}\right)$ [20]. Channel SNR estimates are accurate as long as the packet transmission time is less than the channel coherence time. In IEEE 802.11 based ad hoc networks, for a center frequency of $2.4 \mathrm{GHz}$ and mobility speed of $1 \mathrm{~m} / \mathrm{s}$ $(3.6 \mathrm{~km} / \mathrm{hr})$ the coherence interval is approximately $122.88 \mathrm{~ms}$. For mobility speeds up to $20 \mathrm{~m} / \mathrm{s}$, coherence interval is an order 
of multiple packet transmission times; so channel coherence time is constant for the entire packet transmission. Hence the complex physical model of the wireless channel can be approximated by the protocol model as under.

As per the protocol model, for a presumed distance between nodes the received signal power fluctuates randomly and thus successful reception of a packet is probabilistic. A packet transmitted from sender $x$ is successfully received at receiver $y$ if the $S I N R_{x y} \geq S I N R_{T h}$ given as:

$$
p_{s}=\operatorname{Prob}\left[\frac{s}{N+I} \geq \beta\right]
$$

where $s$ is the received signal strength (RSS), $N$ is the background noise, $I$ is the interference and $\beta$ is the SINR threshold.

Assuming noise to be normally distributed and ignoring the interference, RSS can be taken as a measure of SNR. I.e. two nodes can establish connection if the received signal power is superior than certain predefined threshold $\left(R S S_{x y} \geq R S S_{T h}\right)$.

$$
p_{x y}(c)=\operatorname{Prob}\left[R S S x y \geq \beta^{\prime}\right]
$$

where $p_{x y}(c)$ is the probability of $x$ and $y$ being connected, and $\beta^{\prime}$ represents threshold $\beta$ ignoring noise and interference. Conversely, outage probability (link failure probability) between nodes $x$ and $y$ is:

$$
p_{x y}(o)=\left[1-p_{x y}(c)\right]
$$

The default signal strength threshold (i.e. receiver sensitivity threshold) defines the transmission range of communication and is the limiting radius of circular coverage over which communication is possible. As packet reception is based on this threshold, in mobility scenario if the receiver is located at a distance equal to limiting communication radius frequent link breakages will be experienced.

The route discovery procedure of reactive routing protocols e.g. AODV, DSR, etc. is transparent to signal variations over the physical medium. Thus RREQ forwarding decisions at intermediate node do not take into account signal power experienced at the link/s between itself and its precursor/s. The RREQs received over good or weak links are treated equally. If the selected path to the destination is comprised of weak links/ hops, then during channel fluctuations the possibility of path failure will be high and may inhibit its use for data transfer. Thus, efforts taken for RREQ forwarding will be futile and the source node would have to re-discover the route. Addressing this issue and controlling the broadcast storm is the objective of channel quality based adaptive gossip flooding mechanism for AODV. To meet this objective CQAG-AODV suggests the inclusion of signal quality in the RREQ forwarding mechanism of AODV protocol for selecting good quality links so that robust paths are discovered, as well as a number of RREQ packets forwarded are limited.

\section{CQAG-AODV: Channel Quality based Adaptive Gossip Flooding Mechanism for AODV}

CQAG-AODV is essentially a cross-layer design approach [21] wherein channel quality experienced at the physical layer is utilized to adaptively select RREQ flooding probability. The received signal strength (RSS) observed at the link over which RREQ arrived, is considered a measure of channel quality and used in the calculation of gossip probability. The RREQs are rebroadcast with probability $p=1$ if they are received over strong links, else with lower probabilities. Gossip $(p, 1, x)$ approach similar to Gossip $(p, 1, m)$ suggested in [4] is used where; $x$ is the received signal strength threshold $\left(R S S \_T h\right)$. This means, gossiping comes into play only after 1-hop and when signal strength is below RSS_Th. i.e. Source broadcasts the RREQ with probability one, and intermediate nodes follow RSS based gossip for rebroadcasting the RREQs.

An intermediate node (node other than the intended destination), on receiving RREQ message, instead of blind forwarding, first measures the RSS. If RSS value is above the predefined threshold (RSS > RSS_Th), the node forwards it with probability $p=1$, otherwise uses a gossiping approach in which lower gossip probabilities $(p<1)$, are selected depending on the 
experienced RSS. In other words, an intermediate node certainly forwards RREQs, only if they have been received over a good quality link, else it forwards them with a lower probability. Use of lower gossip probabilities ensures that under hostile channel conditions network is not partitioned and some connectivity is conserved even when most of the links are of poor quality.

For incorporating CQAG-AODV algorithm, modification to threshold $\beta^{\prime}$ of Eq. (4) is suggested. The threshold $\beta^{\prime}$ is modified to $\left(\xi^{*} \beta^{\prime}\right)$ and used in the route discovery procedure (where $\xi$ is some constant). This increased threshold $\left(\xi * \beta^{\prime}\right)$ is for selecting the RREQ rebroadcast probability of intermediate node $j$ that has received RREQ from precursor node $i$, and experienced signal strength is $\mu_{i j}$; i.e. $P_{j}\left(\mu_{i j}\right)=f\left(\xi^{*} \beta^{\prime}\right)$. Whereas $\left(\xi^{*} \beta^{\prime}\right)$ is used only for route discovery, the default threshold $\beta^{\prime}$ is used for data transfer and all other mechanisms. Defining two different thresholds $\beta^{\prime}$ and $\left(\xi^{*} \beta^{\prime}\right)$ in the routing mechanism has a dual objective; it helps to discover better quality paths during the route discovery phase and maintain higher link connectivity throughout the data transfer phase. Due to higher threshold value $\left(\xi * \beta^{\prime}\right)$ used in route discovery procedure the probability of link establishment may be less but the outage probability during data transfer phase will be reduced since its calculation is based on default threshold $\beta^{\prime}$ and not on $\left(\xi^{*} \beta^{\prime}\right)$.

More RREQs a node forwards over a particular hop, higher is its likelihood to be selected for routing and vice-versa. Since CQAG-AODV tunes the rate of RREQ forwarding on the basis of channel quality experienced, higher the value of signal strength of a link/hop along which RREQ is received, larger will be $P_{j}\left(\mu_{i j}\right)$ and vice versa. i.e. By forwarding lesser RREQs over poor signal quality links as against good quality links, CQAG-AODV lessens the probability of discovering weak routes that offer poor signal strength. Thus the majority of routes/paths discovered will be contributed by good signal quality links/hops (better than that with default threshold of $\beta^{\prime}$ ), whose SINR does not fall below acceptable value, even when noise or interference levels rise somewhat above average. Thus CQAG-AODV ensures discovery of robust routes where link failure probability is reduced when channel fluctuations are experienced, and thus maintain higher connectivity throughout the data transfer phase.

\subsection{CQAG-AODV algorithm implementation}

CQAG-AODV is implemented by modifying RFC 3561 based AODV implementation of QualNet simulator. It uses the receiver sensitivity thresholds defined for deciding the MAC data rates of IEEE 802.11b [22] as RSS thresholds for selecting gossip probability $p$ (i.e. the modified threshold $\left(\xi^{*} \beta^{\prime}\right)$ of Eq. (4)). Four different receiver sensitivity thresholds as defined in QualNet simulator are $\{-94 \mathrm{dBm},-91 \mathrm{dBm},-87 \mathrm{dBm}$ and $-83 \mathrm{dBm}\}$. Authors of [23] demonstrated effective use of the receiver sensitivity thresholds to decide optimal MAC transmission rate for improving the network capacity. CQAG-AODV associates different probabilities, drawn from Gaussian distribution, with these thresholds to select best quality hops to improve the connectivity. The RSS values and associated probabilities are presented in Table 1.

Table 1 RSS Based Gossip Probability Selection

\begin{tabular}{ccc}
\hline Received Signal Strength(RSS) Value & Channel /Link Quality & Gossip Probability \\
\hline$R S S \geq-83 \mathrm{dBm}$ & Best & $p=1$ \\
$-87 \mathrm{dBm} \leq R S S<-83 \mathrm{dBm}$ & Good & $p=0.66$ \\
$-91 \mathrm{dBm} \leq R S S<-87 \mathrm{dBm}$ & Weak & $p=0.50$ \\
$-94 \mathrm{dBm} \leq R S S<-91 \mathrm{dBm}$ & Poor & $p=0.33$ \\
$R S S<-94 \mathrm{dBm}$ & Worst & $\mathrm{p}=0$ \\
\hline \hline
\end{tabular}

When an intermediate node receives RREQ, it first measures the RSS value and compares it with a series of receiver sensitivity thresholds (defined in Table 1). If RSS value is below the predefined threshold (RSS_Th $=-95 \mathrm{dBm})$, the intermediate node discards it otherwise selects the forwarding probability on the basis of the RSS value. The flowchart of CQAG-AODV is shown in Fig. 1. 


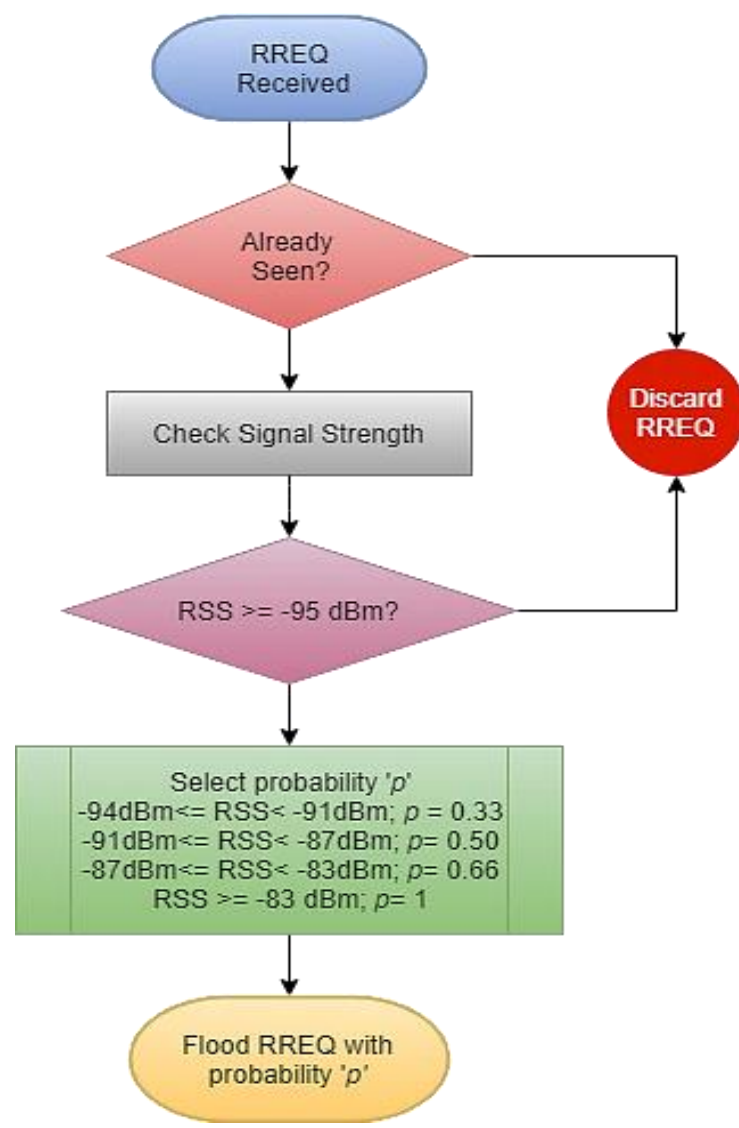

Fig. 1 CQAG-AODV flowchart

Associating RREQ forwarding probability $p=1$ with receiver sensitivity threshold of $-83 \mathrm{dBm}$, ensures that best signal quality paths are discovered with the highest probability. For good quality links, using gossip probability of $p=0.66$ assures the same level of network connectivity although 33\% lesser RREQs are forwarded. This is because $p$ value between 0.65 and 0.75 ensures that almost all nodes in the network will get the messages $\left(\theta_{1}^{S}(0.65)=0.95\right)$ [4]. Additional lower probabilities are used as an effort to circumvent network partitioning, when most of the links are of poor quality. Considering multiple RSS thresholds and using different flooding probabilities, the proposed scheme will perform equally well in scenarios of low as well as high node density and mobility.

Incorporating the proposed CQAG-AODV algorithm in AODV is quite simple and practicable, as it does not need any changes either to the control packet formats or routing table structure of standard AODV or IEEE 802.11 implementation. As discussed earlier, CQAG-AODV only suggests changes to the route discovery mechanism (Fig. 1), where multiple RSS thresholds are used for deciding the RREQ forwarding rate. Also, no significant delay will be added due to modified RREQ forwarding logic, since only three additional RSS comparison operations of time complexity $O(1)$ are involved. Taking into account the clock frequency $(0.8-2 \mathrm{GHz})$ of ARM Cortex processors that are widely used in IEEE 802.11 compliant mobile devices, the processing time for these comparisons will be $<1 \mu \mathrm{s}$. Thus CQAG-AODV will not incur any additional overhead or processing delay as compared to conventional AODV.

\section{CQAG-AODV Path Selection and Connectivity: Analytical Treatment}

Since CQAG-AODV follows signal strength based gossiping approach, wherein the RREQs are forwarded with varying probabilities, the existence of an edge (link/hop) between neighboring nodes $i$ and $j$ are probabilistic with probability $p_{i j}$. It is known from the results of percolation theory that gossiping exhibits bimodal behavior [24]; the gossip reaches to almost all nodes resulting in a connected graph, only when the gossip probability $p$ is greater than or equal to some critical probability $p_{c}$. (i.e. $\theta(p)>0$ ), or it does not reach any node (i.e. $\theta(p)=0$ ). 
The aim of this paper is to determine the connectivity of CQAG-AODV, as well as to determine whether CQAG-AODV actually discovers and selects better quality paths. This section tries to answer two fundamental questions:

i. Given a network consisting of many paths from source to destination, what is the probability that a source $S$ is connected to destination $D$, via a particular path $R_{x}$, from the available $r$ paths?

ii. What is the probability that the ad hoc network (graph) is connected, when the links (edges) joining the nodes (vertices) occur with some probability?

The first subsection models and illustrates how CQAG-AODV discovers best quality paths, and the network connectivity of CQAG-AODV is presented in the next subsection.

\subsection{CQAG-AODV best quality path selection}

During the route discovery phase, based on the RSS value of arriving RREQ packets intermediate nodes rebroadcast them independently with probability, that takes one of the four values from $\left\{p_{\text {best }}, p_{\text {good }}, p_{\text {weak }}, p_{\text {poor }}\right\}$ as defined in Table 1 . For a given network, if there are $r$ possible paths/routes between given source-destination $(S-D)$ pair, then we are interested in finding the probability that RREQ reaches the destination traversing the best path from available $r$ paths.

The probability that RREQ originated from source can reach the destination, traversing one of the $r$ paths can be represented as:

$$
P(S \rightarrow D)=P\left(R_{1} \cup R_{2} \cup R_{3} \ldots \cup R_{r}\right)
$$

i.e.

$$
P(S \rightarrow D)=1-\prod_{1}^{r}\left(1-p_{r}\right)
$$

where $p_{r}$ is the probability that RREQ reaches the destination along route $R_{r}$; and $\left(1-p_{r}\right)$ is the probability that RREQ can't reach the destination along any of the paths.

For a given path, if source and destination are separated by ' $h$ ' hops, then the probability that RREQ can reach the destination along the $r^{\text {th }}$ path can be found using the independence property [25] as:

$$
p_{R_{r}}=\left\{p_{1} * p_{2} * \ldots * p_{h}\right\}=\prod_{1}^{h} p_{i}
$$

where $p_{R_{r}}$ represents the probability of existence of $r^{\text {th }}$ path and $p_{i}$ is the probability of existence of the $i^{\text {th }}$ hop.

Out of the $r$ available paths/routes, CQAG-AODV is expected to select the route comprising of best signal quality hops, with maximum probability. The probability that best quality path is selected out of the available $r$ paths can be expressed as:

$$
P(\text { best quality path })=\max \left\{p_{r}\right\}, \quad r=R_{1} \text { to } R_{r}
$$

Preposition 1: CQAG-AODV discovers better channel quality paths and thus results in a connected network comprising of the best quality route with maximum probability.

Let $r^{\text {th }}$ path is the best path in terms of signal quality; then it is expected that the proposed algorithm should select this path with higher probability. We apply the notion of Certainty Factor (CF) of expert systems [26] to determine the certainty level of our expectation.

Here the premise is route/path between source and destination exists with probability $\mathrm{P}(\mathrm{S} \rightarrow \mathrm{D})$.

$$
\text { i.e. } C F[\operatorname{path}(S \rightarrow D)]=P(S \rightarrow D)
$$


and following rules are applied:

Rule1: IF path_exists $(\mathrm{S} \rightarrow \mathrm{D})$ THEN has_route $\mathrm{R}_{\mathrm{r}}(\mathrm{S} \rightarrow \mathrm{D})$ with $\mathrm{CF}=p_{R_{r}}$

Rule2: IF has_route $\mathrm{R}_{\mathrm{r}}(\mathrm{S} \rightarrow \mathrm{D}) \mathrm{THEN}$ route $\mathrm{R}_{\mathrm{r}}(\mathrm{S} \rightarrow \mathrm{D})$ _selected with $\mathrm{CF}=1 / \mathrm{r}$

then combining the premise and rules, it yields

$$
\begin{aligned}
& \mathrm{CF}\left[\text { path_ } \mathrm{R}_{\mathrm{r}} \text { exists }(\mathrm{S} \rightarrow \mathrm{D})\right]=\mathrm{CF}[\text { path }(\mathrm{S} \rightarrow \mathrm{D})] * \mathrm{CF}(\text { Rule } 1) \\
& \mathrm{CF}\left[\text { path_ } \mathrm{R}_{\mathrm{r}} \text { selected }(\mathrm{S} \rightarrow \mathrm{D})\right]=\mathrm{CF}[\text { path }(\mathrm{S} \rightarrow \mathrm{D})] * \mathrm{CF}(\text { Rule1 }) * \mathrm{CF}(\text { Rule } 2)=P(S \rightarrow D) * p_{R r_{r}} * \frac{1}{r}
\end{aligned}
$$

Calculating the CF of all possible paths between $S-D$, if we get highest CF for the path with best channel quality, then we can say CQAG-AODV discovers best quality paths more often. Next we verify the same considering an example scenario.

\subsection{CQAG-AODV quality path discovery illustration}

To illustrate the proof of concept, we consider a particular case of a simple ad hoc network consisting of 10 nodes placed as shown in Fig. 2. Here, circles represent the nodes; $S$ being the source, $D$ the destination, and $A$ through $I$ the intermediate forwarding nodes. The wireless links between the nodes represented by dotted lines depict these nodes are in coverage distance (transmission range) of each other; with the link quality ( $R S S$ value) marked on top. CQAG-AODV working is illustrated as follows. When source $S$ has a packet for destination $D$ to which route is not readily available in its routing table, $S$ initiates route discovery procedure by broadcasting RREQ packet. Intermediate nodes forward this RREQ with gossip probability decided on the basis of the channel quality (RSS value) of the link over which the RREQ was received. Since CQAG-AODV follows gossiping approach, even though a link may exist between nodes, RREQ forwarding will be probabilistic. Thus, we can say that the existence of link/ hop for packet forwarding is probabilistic.

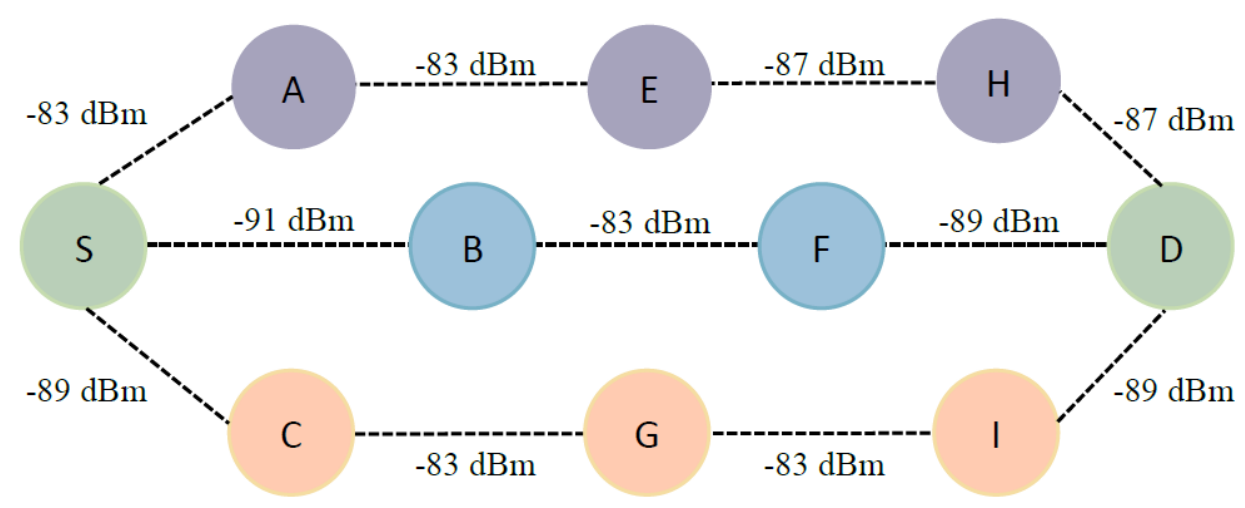

Fig. 2 Simple ad hoc network scenario

In the network of Fig. 2, there are three possible paths; Path 1: S-A-E-H-D, Path 2: S-B-F-D and Path 3: S-C-G-I-D with average $R S S$ value of $-85.22 \mathrm{dBm},-87.53 \mathrm{dBm}$ and $-85.5 \mathrm{dBm}$ respectively. The proposed CQAG-AODV is expected to discover the strongest path i.e. $S-A-E-H-D$.

Referring Table 1, per hop forwarding probabilities at respective hops for the possible paths can be given as: $S-A-E-H-D$ $\{1,1,1,0.66\}, S-B-F-D\{1,1,0.5\}$ and $S-C-G-I-D\{1,0.5,1,1\}$.

Thus probability of RREQ reaching the destination, taking a particular path can be found using the independence property of Eq. (8) as:

$$
\begin{aligned}
& \mathrm{P}(\text { Path } 1)=\mathrm{p} 1=1.0 * 1.0 * 1.0 * 0.66=0.66 \\
& \mathrm{P}(\text { Path } 2)=\mathrm{p} 2=1.0 * 0.5 * 1.0=0.5 \\
& \mathrm{P}(\text { Path } 3)=\mathrm{p} 3=1.0 * 0.5 * 1.0 * 1.0=0.5
\end{aligned}
$$


We then calculate the probability that RREQ can reach the destination traversing one of these paths/routes. i.e. probability of discovering (having) route from $S \rightarrow D$, according to Eq. (7) will be:

$$
P(S \rightarrow D)=1-\prod_{1}^{r}\left(1-p_{r}\right)=1-[0.34 * 0.5 * 0.5]=0.915
$$

thus, $C F[$ path $(S \rightarrow D)]=P(S \rightarrow D)=0.915$

Applying CF Rule of Eq. (13), we have;

$$
C F[\text { path_Rr selected }(S \rightarrow D)]=C F[\text { path }(S \rightarrow D)]^{*} C F(\text { Rule } 1)^{*} C F(\text { Rule2 })=P(S \rightarrow D) * p_{R_{r}} * \frac{1}{r}
$$

For path 1; CF $[$ path_R1 selected $(S \rightarrow D)]=0.915 * 0.66 * 0.33=0.2$

Similarly,

For path 2; CF [path_R2 selected $(S \rightarrow D)]=0.915 * 0.5 * 0.33=0.15$

For path 3; $C F[$ path_R3 selected $(S \rightarrow D)]=0.915 * 0.5 * 0.33=0.15$

From above calculations it is clear that, probability of RREQ originated from source $S$ reaching destination $D$ is maximum for the stronger path; i.e., via Path $1[S-A-E-H-D]$, even though it is longer than Path 2 . The CF calculations indicate that the best quality path $[S-A-E-H-D]$ will be selected, since this path has highest certainty factor $(\mathrm{CF}=0.2)$ as compared to other available paths. This shows CQAG-AODV will discover strongest path with highest probability. Hence our notion that most of the discovered paths by CQAG-AODV will be of good channel quality is justified.

\subsection{Connectivity of $C Q A G-A O D V$}

For given random spatial distribution of nodes and probabilistic radio link model, at given instant of time the network topology can be represented as a geometric random graph $G=G\left(r_{0}, n\right)$, where $n$ represents vertices (nodes) and $r_{0}$ is the transmission range of nodes. We are interested in finding the probability that the network (graph) being connected, when existence of links (edges) connecting the nodes (vertices) is probabilistic. When edge between nodes exists independently with some probability $p$, then the probability of graph being connected is given in [26] as:

$$
P_{c o n}=1-n q^{n-1}
$$

where, $P_{c o n}$ represents probability of graph connectivity, $n=$ number of vertices (nodes), and $q=(1-p)$ is probability that edge does not exist.

The graph (network) is said to be surely connected if $P_{c o n} \geq 0.95$ [27].

\subsection{CQAG-AODV connectivity illustration}

To illustrate the connectivity of CQAG-AODV, let's consider the same particular case of simple ad hoc network as in Fig. 2. As discussed in previous sections, in the route discovery of CQAG-AODV, RREQs are flooded following adaptive gossip approach, where the gossip probability is decided on the basis of channel quality. Thus, here the existence (discovery) of links/hops between nodes is probabilistic, with probability taking one of the four values $\{1,0.66 .0 .5$. 0.33\}, that depends on observed channel quality. For finding the probability of network connectivity, we consider the average of these probability values as edge existence probability ( $p$ ) and use in Eq. (14).

For the network of Fig. 2, where $n=10, P_{c o n}=1-10 *(0.3775)^{9}=0.998$. Since, here $P_{c o n}>0.95$, we can conclude that CQAG-AODV results in a connected network. 


\section{Simulation-based Analysis}

In this section we analyze the connectivity and end-to-end performance of CQAG-AODV through simulation. The objective is to determine how well the proposed algorithm fares as compared to other routing approaches, in realistic network setup consisting a fairly large number of nodes. The analysis is carried out considering static as well as mobile networks. Static network simulation presents the connectivity analysis in an explicit manner, whereas in the mobile network scenario the analysis is based on end-to-end performance. Next subsections present performance analysis of CQAG-AODV in static and mobile network scenarios.

\subsection{CQAG-AODV performance in static scenario}

In this section, we find the probability of ad hoc network consisting of $n$ nodes being connected when each node has a transmission range $r_{0}$. The connectivity analysis of [28] suggested that, for given node placement, the network is connected if $r_{0} \geq r_{t h}$ (i.e longest link in the minimum spanning tree is the minimum range). Very small values of $n$ and/or $r_{0}$ result in a disconnected network, and as $n$ or $r_{0}$ or both increases, the probability of connectivity increases. The range should be large enough to keep the network connected, at the same time small enough to render low interference between nodes and lesser power consumption. Following this analysis, we analyzed the network connectivity as a function of transmission range $r_{0}$ when the number of nodes in the network is fixed.
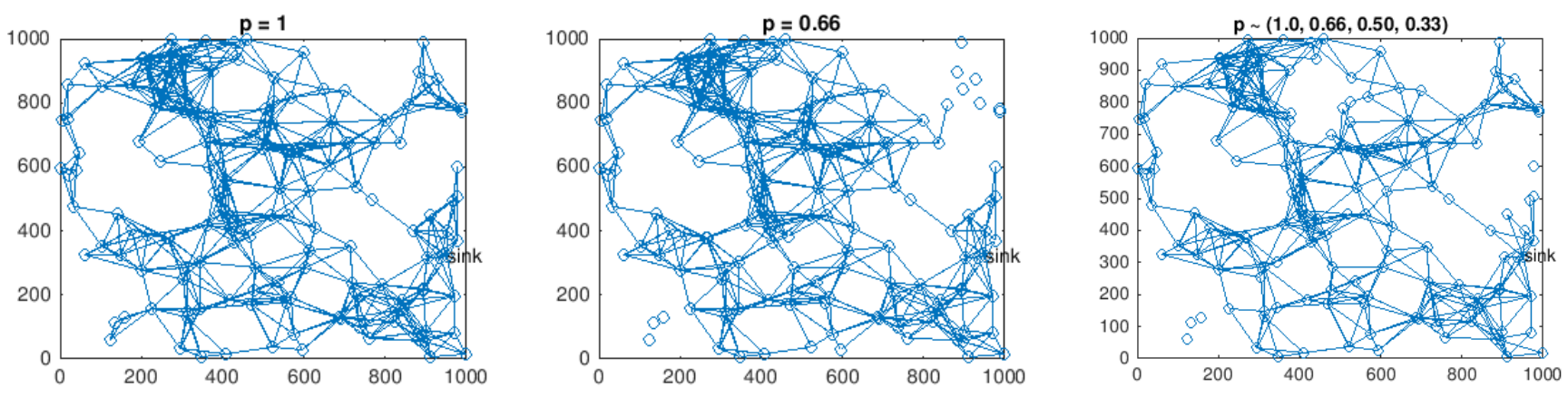

(a) Network Connectivity for $r_{0}=150 \mathrm{~m}$
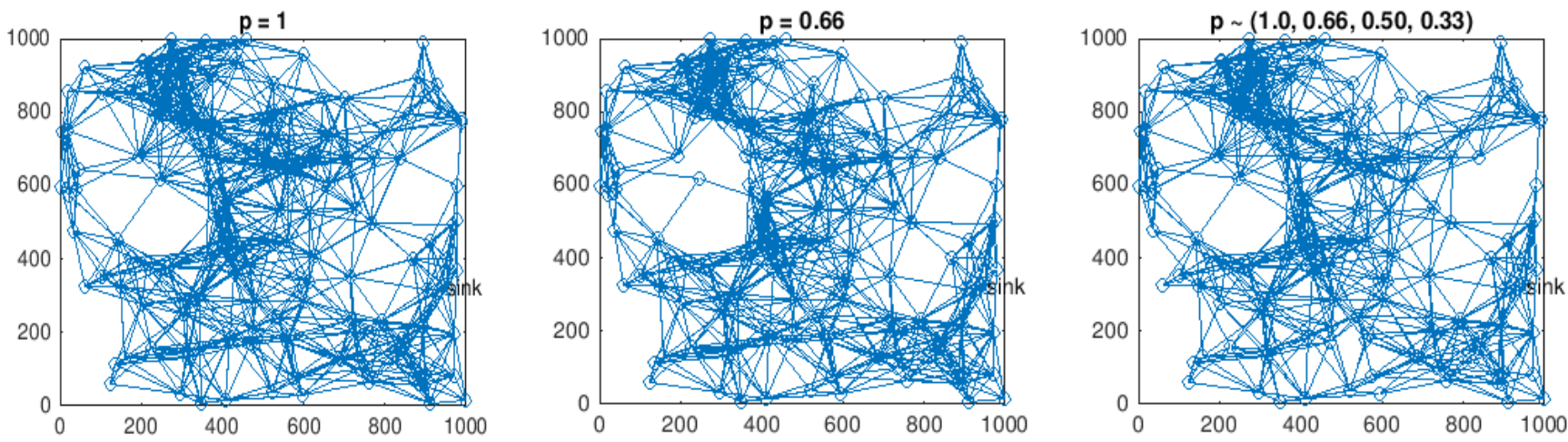

(b) Network Connectivity for $r_{0}=200 \mathrm{~m}$

Fig. 3 Network Connectivity with different flooding probability $(p)$ values

Consider the placement of $n$ nodes generated by uniform random distribution on a square area. A static network consisting of 150 nodes uniformly distributed over $1000 \mathrm{~m} * 1000 \mathrm{~m}$ terrain, with a single source-destination pair is simulated in Matlab, that represents a geometric random graph $G\left(r_{0}, n\right)$. Initially, the transmission range of all nodes is set to $125 \mathrm{~m}$ and then gradually increased so that links will be added to the network. Thus, at some point of time transmission range, $r_{0}$ will be sufficiently large to make $G\left(r_{0}, n\right)$ connected. It is expected that although CQAG-AODV controls the RREQ rebroadcasts, it should maintain same level of connectivity as AODV. Fig. 3 presents connectivity diagrams for different transmission range $\left(r_{0}\right)$ and flooding probabilities $(p)$. Here, diagram for $p=1$ represents connectivity achieved with flooding based route 
discovery of conventional AODV, the connectivity of gossip-based AODV $+\mathrm{G}$ is shown by $p=0.66$, and the graph with $p=(1$, $0.66,0.5,0.33)$, illustrates the connectivity of channel quality based flooding mechanism of CQAG-AODV. From the connectivity diagrams, it can be concluded that, although the probabilistic flooding algorithms; AODV+G and CQAG-AODV, flood lesser number of RREQs, they offer the same level of connectivity as AODV when transmission range is sufficiently large.



(a) Connectivity Vs Node Coverage Range

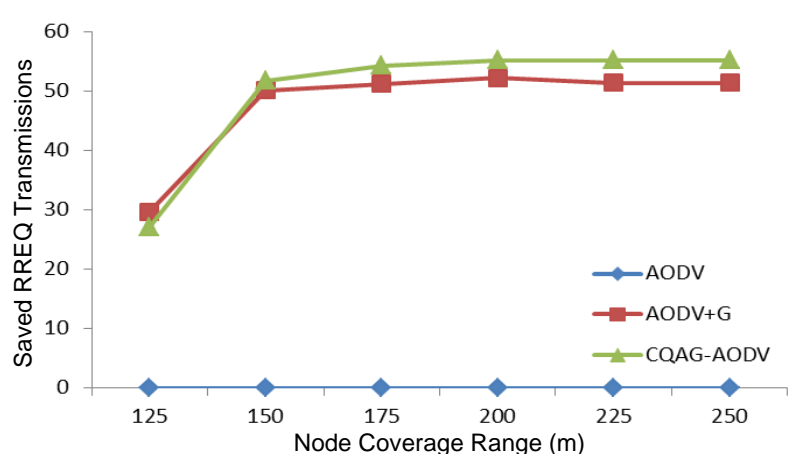

(b) Saved RREQ Transmissions Vs Node Coverage Range

Fig. 4 Connectivity and Saved RREQ Transmissions in the static scenario

It can be seen from Fig. 4 (a) that, for all coverage distances routing mechanism of AODV results in a surely connected network (connectivity probability of 1). Whereas, for coverage distances less than $150 \mathrm{~m}$, the connectivity of both probabilistic algorithms AODV+G and CQAG-AODV is poor, since for this lower coverage distance, the RREQ rebroadcasts of lower probability may have resulted in a partitioned network. For coverage distances above $150 \mathrm{~m}$, CQAG-AODV delivers similar performance as that of conventional AODV in terms of network connectivity, requiring lesser number of RREQ retransmissions as compared to AODV (Fig. 4 (b)).

Because, increasing transmission range gradually, will result in increased signal strength experienced, and thus selection of higher gossip probability (tending to 0.66 ) for RREQ rebroadcasts. Since the numbers of RREQ rebroadcasts will still be lower than flooding, CQAG-AODV rebroadcasts a lesser number of RREQs than AODV and thus results in significantly saved RREQ retransmissions. However, the connectivity and saved RREQ transmissions of CQAG-AODV is comparable to AODV + G. Hence it can be concluded that by tuning the gossip probability with different signal strength thresholds, CQAG-AODV can control the RREQ rebroadcasts and check the broadcast storm without adversely affecting network connectivity when the coverage distance of nodes is sufficiently large.

\subsection{CQAG-AODV performance in mobility scenario}

To analyze the performance of CQAG-AODV in mobile network scenario, a medium-sized ad hoc network consisting of 150 mobile nodes uniformly distributed in a square terrain is simulated in QualNet simulator. 10 Constant Bit Rate (CBR) connections are set between randomly selected source-destination pairs. Each CBR connection corresponds to a traffic rate of 4 packets/sec. The nodes move following an RWP mobility model with pause time 0s and minimum node speed of $1 \mathrm{~m} / \mathrm{s}$. Two set of experiments were designed to represent an ad hoc network with dense and sparse node density. For representing dense and sparse networks, we selected two different terrain dimensions; $1000 \mathrm{~m}^{2}$ and $2500 \mathrm{~m}^{2}$, resulting in node densities of 15 nodes $/ 10 \mathrm{~m}^{2}$ and 6 nodes $/ 10 \mathrm{~m}^{2}$ respectively. Since network connectivity is a function of node degree [28], it becomes important to consider these diverse network scenarios. For evaluating the performance, mobility speed of nodes is varied from $1.25 \mathrm{~m} / \mathrm{s}$ to very high speeds up to $40 \mathrm{~m} / \mathrm{s}$. The speed is initially set to the lowest value of $1.25 \mathrm{~m} / \mathrm{s}$ and doubled every time up to $10 \mathrm{~m} / \mathrm{s}$, thereafter it is gradually increased by $5 \mathrm{~m} / \mathrm{s}$ up to $40 \mathrm{~m} / \mathrm{s}$. The objective is to analyze the ability of CQAG-AODV to maintain connectivity when channel quality fluctuations arise due to node mobility. Table 2 presents detailed simulation parameters. 
Table 2 RSS Based Gossip Probability Selection

\begin{tabular}{|c|c|}
\hline Parameter & Value \\
\hline No. of Nodes & 150 \\
\hline Area & $1000 \mathrm{~m} * 1000 \mathrm{~m}$ and $2500 \mathrm{~m} * 2500 \mathrm{~m}$ \\
\hline Node Placement Strategy & Uniform \\
\hline Simulation Time & $180 \mathrm{sec}$ \\
\hline Channel Frequency & $2.4 \mathrm{GHz}$ \\
\hline Path Loss Model/ Fading model & Two ray Model/ None \\
\hline Propagation Limit & $-95 \mathrm{dBm}$ \\
\hline Mobility Model & RWP, pause time $=0 \mathrm{~s}$ \\
\hline Mobility speed (m/s) & $1.25,2.5,5.0,10,15,20,25,30,35,40$ \\
\hline PHY / MAC Layer Protocol & IEEE $802.11 \mathrm{~b}$ \\
\hline No. of Traffic Flows & 10 CBR connections \\
\hline Payload size & 512 bytes \\
\hline Traffic Sending Rate & 4 packets/sec (i.e $16 \mathrm{kbps})$ \\
\hline
\end{tabular}

Performance of CQAG-AODV is evaluated on the metrics of Packet Delivery Ratio (PDR), throughput, routing overhead (RREQ forwarding overhead) and link breakages, and compared with conventional AODV and gossip-based AODV+G.

A. Performance in dense network scenario

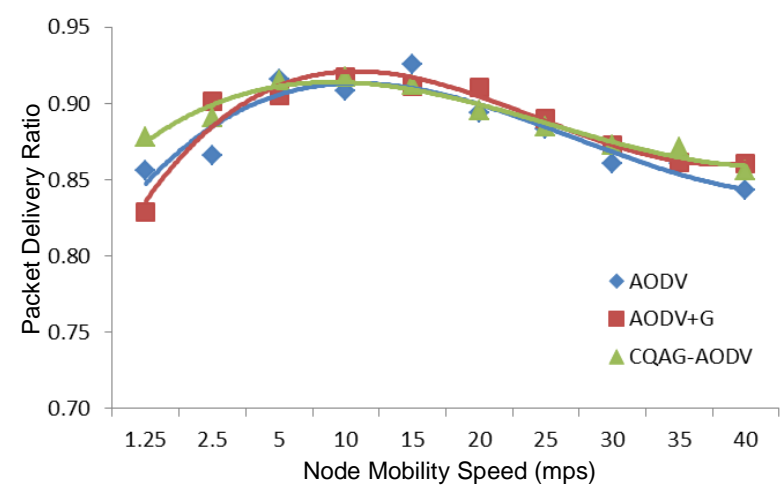

(a) PDR Vs Mobility Speed

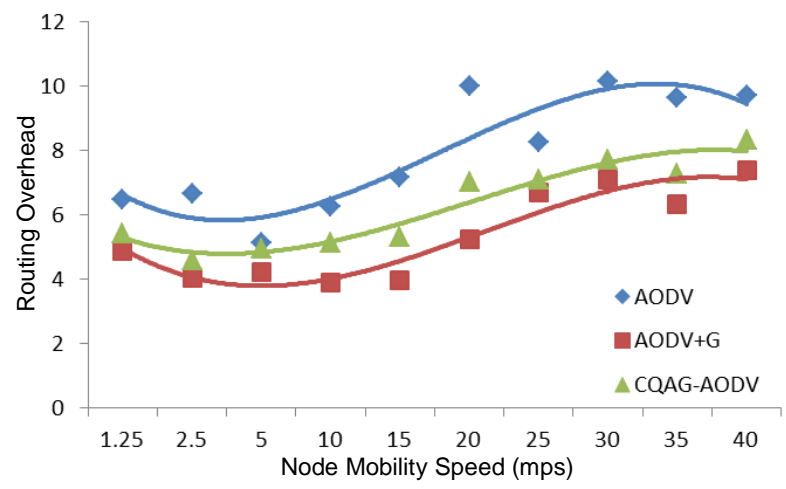

(c) Routing Overhead Vs Mobility Speed

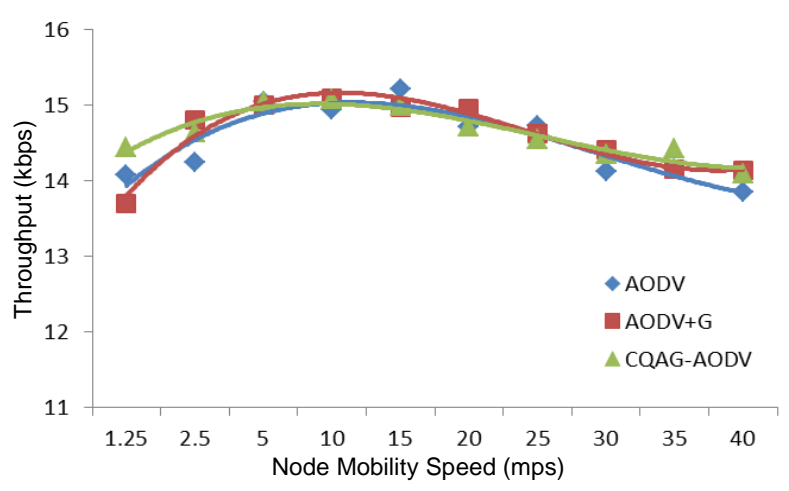

(b) Throughput Vs Mobility Speed

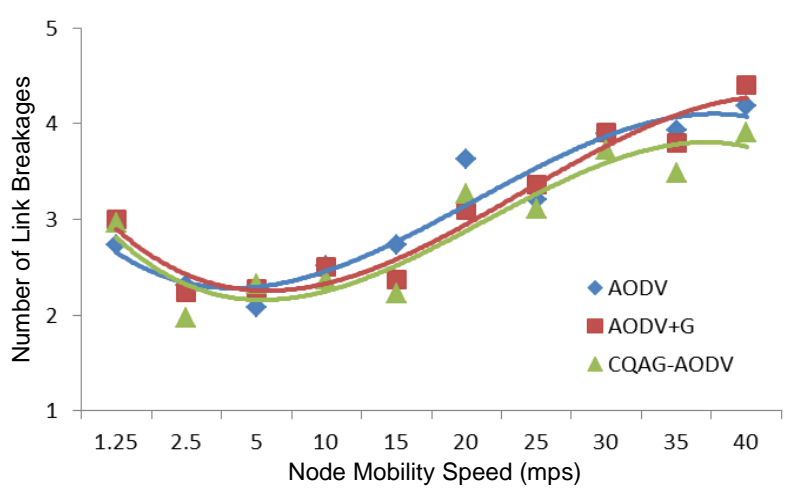

(d) Link Breakages Vs Mobility Speed

Fig. 5 Performance Analysis of CQAG-AODV for Node Mobility in Dense Network scenario

The objective is to analyze how CQAG-AODV performs when network is densely populated. Here 150 nodes are placed in a terrain of $1000 \mathrm{~m}^{2}$. Fig. 5 shows the results of different performance metrics. It is expected that CQAG-AODV should reduce the RREQ broadcast and link breakages, at the same time maintain the same level of connectivity as conventional AODV and AODV+G. It can be seen from Figs. 5 (a) and (d) that CQAG-AODV incurs $21 \%$ lesser routing overhead as compared to AODV but still delivers the same average PDR and throughput as that of conventional AODV and Gossip AODV+G. This implicitly confirms that network connectivity achieved with CQAG-AODV is comparable with AODV and AODV + G. However, the routing overhead of CQAG-AODV is $\sim 17 \%$ more as compared to AODV+G. This is the result of fixed gossip probability $(p=0.66)$ used in AODV $+\mathrm{G}$ for RREQ rebroadcasting, which saves more RREQs than 
CQAG-AODV's RSS based adaptive gossip mechanism. In this network setup as the node density is higher, nodes are closer and hence most of the times the channel quality is best or good, so CQAG-AODV gossips with higher probability ( $p=1$ or 0.66 ), whereas $\mathrm{AODV}+\mathrm{G}$ uses $p=0.66$ at all times and thus incurs lowest routing overhead.

The average link breakages experienced with CQAG-AODV are lesser by $6 \%$ and $5 \%$ as compared to AODV and $\mathrm{AODV}+\mathrm{G}$ respectively. This is because CQAG-AODV discovers better quality paths/routes as against the arbitrary paths discovered by AODV and AODV+G. As CQAG-AODV accounts higher RSS value than the default threshold in the route discovery decision, routes discovered with CQAG-AODV can sustain the channel fluctuations arising from node mobility; hence failures experienced in the discovered path are lesser. Whereas in conventional routing approaches channel quality is not accounted in the route discovery, thus discovered path will be contributed by weak links that experience frequent breakages.

Moreover, as can be seen from Figs. 5 (a) and (b), CQAG-AODV achieves some improvement in end-to-end performance over the other two approaches, only for very low and very high mobility speeds $(<2.5$ and $>25$ mps $)$. At these speeds, the nodes that are at the boundary of limiting communication radius, often move out of coverage of their neighbors, and hence the performance of AODV and AODV $+\mathrm{G}$ is degraded.

B. Performance in sparse network scenario

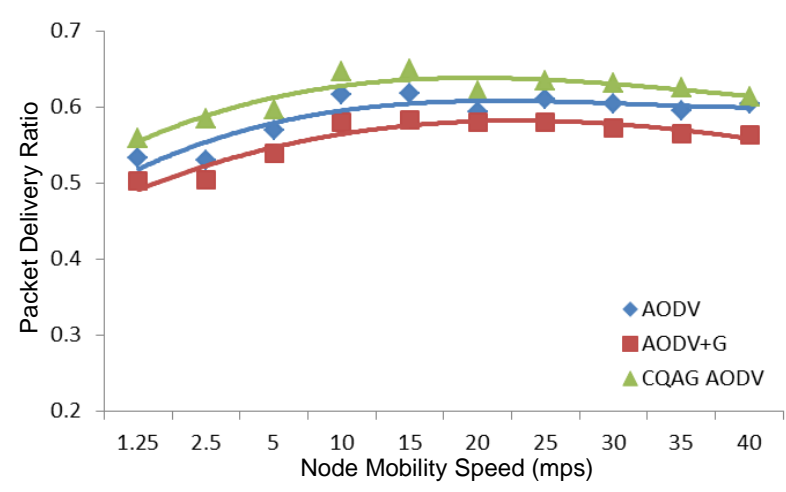

(a) PDR Vs Mobility Speed

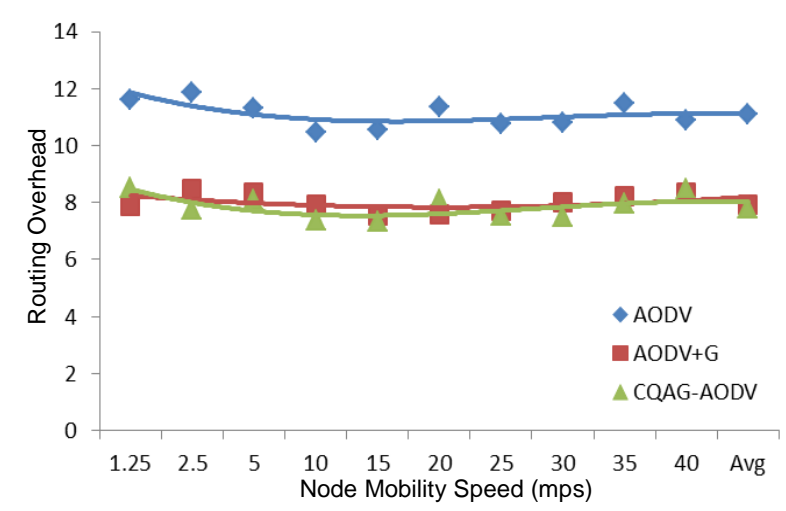

(c) Routing Overhead Vs Mobility Speed

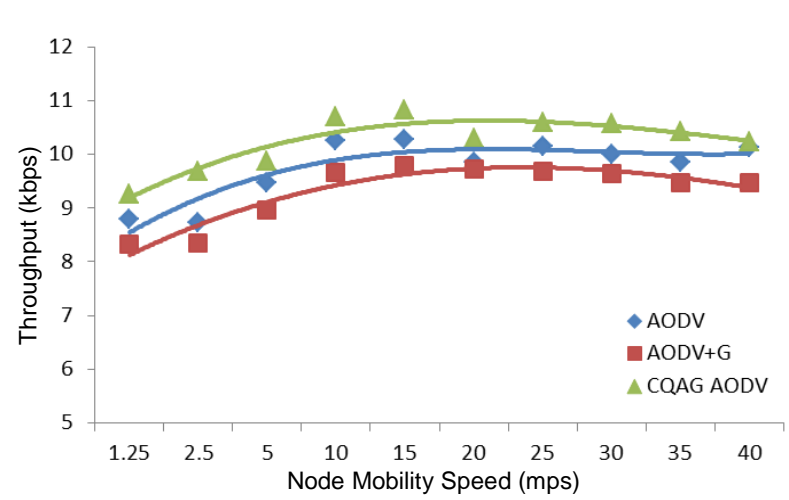

(b) Throughput Vs Mobility Speed

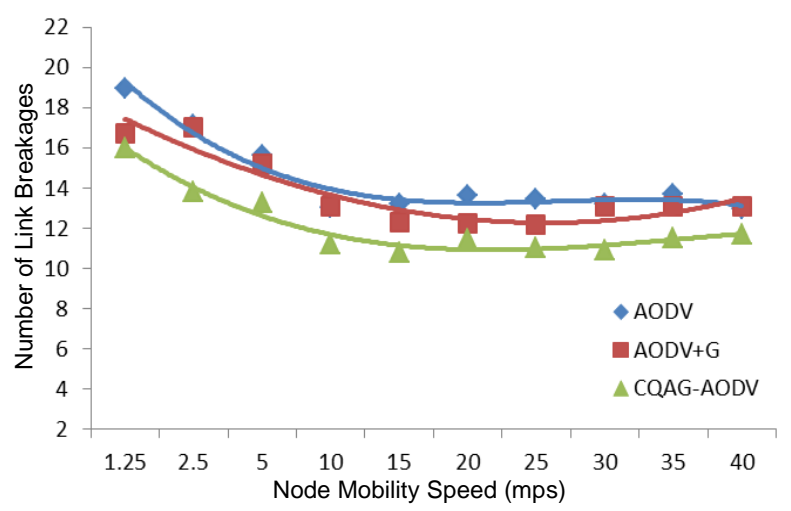

(d) Link Breakages Vs Mobility Speed

Fig. 6 Performance Analysis of CQAG-AODV for Node Mobility in Sparse Network scenario

Here we analyze the performance of CQAG-AODV in the scenario where the nodes per unit area are less- 150 nodes placed in a terrain of $2500 \mathrm{~m}^{2}$. Since node density is less, the inter node distance is more, and thus links contributing to the multi-hop network will be weak and experience failures, thereby reducing the end-to-end performance. Hence, in this network setup, due to its signal quality-based route discovery mechanism, CQAG-AODV will discover robust paths contributed by better quality links and deliver improved performance than AODV and AODV+G.

As can be seen from plots of Fig. 6, CQAG-AODV beats AODV and AODV+G on all performance metrics. The average link breakages experienced with CQAG-AODV are lesser by $16 \%$ and $12 \%$ as compared to AODV and AODV+G respectively. 
CQAG-AODV incurs 29\% lesser routing overhead to deliver 5\% higher average PDR and throughput than conventional AODV. Although, as compared to AODV $+\mathrm{G}$ routing overhead of CQAG-AODV is reduced merely by $2 \%$, its PDR and throughput show significant improvement of $10 \%$ over AODV+G. This shows the fixed probability gossip-based route discovery of $A O D V+G$ saves the RREQ broadcast but at the cost of degraded end-to-end performance, whereas CQAG-AODV does not compromise on end-to-end performance.

The reason for the degraded performance of AODV $+\mathrm{G}$ as compared to AODV and CQAG-AODV lies in reduced node density. It may happen that, during route discovery process the probabilistic RREQ forwarding of AODV+G may not converge to successful route discovery, or RREQs will reach to destination after forwarded by intermediate nodes that are far away from each other (i.e. at the boundary of coverage). Due to mobility, the discovered path may not be useful for sending route replies or it cannot be sustained longer for data transfer, as the links/hops (that are already weak) contributing to the route may fail soon.

\subsection{CQAG-AODV energy performance}

The connectivity an analysis proved that probabilistic approaches controlled the routing overhead without affecting network connectivity. It is affirmed through performance evaluation that in dense network scenario PDR of all routing approaches is almost the same. Whereas in sparse network scenario, as compared to AODV, CQAG-AODV delivers $5 \%$ more PDR, while the PDR of AODV+G is degraded by 5\%. CQAG-AODV and AODV+G incur $\sim 21 \%$ and $48 \%$ less routing overhead as compared to AODV when network is dense; whereas in sparse network scenario, this reduction is $\sim 29 \%$. Thus, it can be expected that as the RREQ broadcasts are saved, these schemes will conserve node energy. This section investigates the energy conservation capability of these proposals.

The energy conservation achieved is analyzed on the metric of normalized energy consumption per successful packet reception; defined as the ratio of energy consumed by the nodes and number of packets received successfully. For analyzing the energy performance, the same simulation scenario of Table 2 is considered with an initial energy of nodes set to 2 mAh. Performance is evaluated by varying the node mobility speed, as was carried out in the previous sub-section. Figure 7 (a) and (b) present plots of normalized energy consumption per successful packet reception for dense and sparse network scenarios.

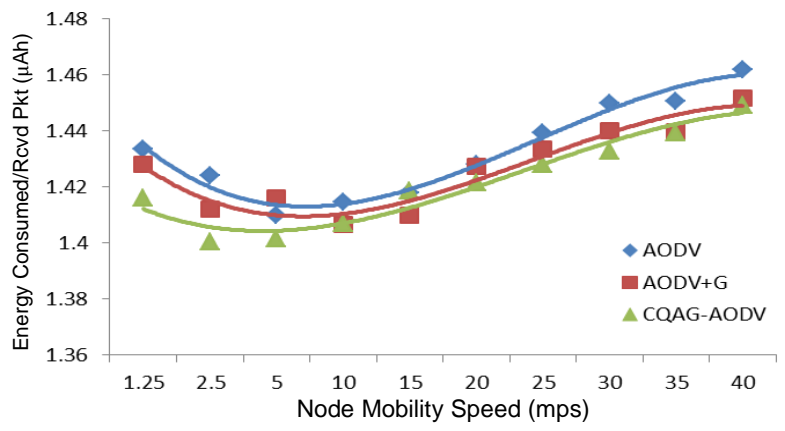

(a) Normalized Energy Consumption per Successful Packet Reception in Dense Network

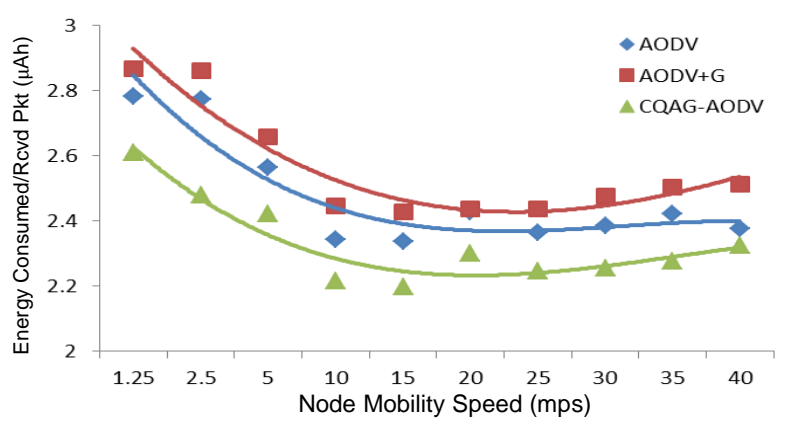

(b) Normalized Energy Consumption per Successful Packet Reception in Sparse Network

Fig. 7 Energy Performance of CQAG-AODV for Node Mobility in different Network scenarios

It can be observed that in dense network scenario both probabilistic approaches (CQAG-AODV and AODV+G) result in lesser normalized energy consumption than AODV. This is obvious because the probabilistic flooding mechanism adopted in these schemes reduces the number of RREQs forwarded and thus conserves node energy. Since the network is densely populated; even though fewer RREQs are flooded, the network will not be partitioned as every node can easily find a forwarder and establish a path to a destination.

However, in sparse network scenario, this is not the case. Since node density is lesser, the low probability broadcasts may end up in a void situation with no nodes forwarding the RREQs, and thus result in a partitioned network. Moreover, as the links 
contributing to the discovered route will be weak, link breakages experienced will be higher. Thus as compared to AODV, AODV+G delivers lesser PDR incurring $\sim 3.5 \%$ more normalized energy consumption. Whereas the channel quality based adaptive gossip flooding mechanism of CQAG-AODV discovers strong routes that are robust to failure to offer higher PDR as compared to AODV and thus incurring 6\% lesser normalized energy consumption.

From this analysis it is clear that CQAG-AODV exhibits upper-edge over AODV+G since it delivers the same performance as conventional AODV incurring reduced energy consumption; both in dense as well as sparse networks. Thus, CQAG-AODV proves its usability as an energy optimized routing approach that can improve the node/network lifetime, as achieved with [28-29]. However, as node energy is not accounted for routing decisions, it is not able to salvage energy-critical nodes.

\section{Conclusions}

This paper presented a connectivity analysis of Channel Quality-based Adaptive Gossip flooding mechanism for AODV (CQAG-AODV), which was proposed to address "broadcast storm" problem. It is basically a cross layer design approach that exploits channel quality (RSS) at the physical layer to adaptively decide the RREQ flooding probability. The proposed algorithm discovers better signal quality paths by forwarding more RREQs over strong links as against weak links, and thus improves routing performance. The theoretical analysis proved that CQAG-AODV algorithm discovers best paths/routes from all available paths without affecting network connectivity.

Simulation results show that by reducing the number of RREQ rebroadcasts CQAG-AODV addressed the "broadcast storm" problem. Moreover, as the discovered routes are of good signal quality, link/path failures experienced in CQAG-AODV are reduced. CQAG-AODV offered the same level of connectivity and end to-to-end performance as AODV and $A O D V+G$ in dense network scenario, whereas it outperformed both AODV as well as AODV+G on all performance metrics (PDR, throughput, routing overhead, and link breakages experienced), in a sparsely populated network. CQAG-AODV delivered 5\% more PDR and throughput than AODV by incurring 29\% less routing overhead. Whereas, as compared to $\mathrm{AODV}+\mathrm{G}$ the routing overhead of CQAG-AODV is merely $2 \%$ lesser, it delivers $10 \%$ more PDR and throughput than AODV+G. Further, the improvement in PDR is achieved at the cost of $\sim 6 \%$ lesser normalized energy consumption as compared to AODV. Thus, channel quality based adaptive gossip flooding mechanism of CQAG-AODV is an effective proposition for controlling broadcast storm as well as maintaining the connectivity so as to assure QoS and energy optimization in diverse network scenarios.

\section{Conflicts of Interest}

The authors declare no conflict of interest.

\section{References}

[1] C. E. Perkins, S. Das, “Ad Hoc on-demand distance vector (AODV) routing,” RFC 3561, July 2003.

[2] YC Tseng, SY. Ni, YS. Chen and JP. Sheu, "The broadcast storm problem in a mobile ad hoc network," Wireless Networks, vol.8, no.2-3, pp. 153-167, March. 2002.

[3] O. Tonguz, N. Wisitpongphan, J. Parikh, F. Bai, P. Mudalige and V. Sadekar, "On the broadcast storm problem in ad hoc wireless networks," Proc. 3rd International Conference on Broadcast Communications, Networks and Systems (BROADNETS), Octorber 2006, pp. 1-11.

[4] Z. Haas, J. Y. Halpern, and L. Li, “Gossip-based ad hoc routing,” IEEE/ACM Transactions on Networking, vol. 14, no. 3, pp.479-491, June 2006.

[5] B. Blywis, M. Güneş, F. Juraschek, and S. Hofmann, "Gossip routing in wireless mesh networks," Proc. 21st IEEE International Symposium on Personal Indoor and Mobile Radio Communications (PIMRC), September 2010, pp. $1572-1577$. 
[6] X. M. Zhang, E.B. Wang, J. J. Xia, and D. K. Sung, “A neighbor coverage based probabilistic rebroadcast for reducing routing overhead in mobile ad hoc networks," IEEE Transactions on Mobile Computing, vol. 13, no.3, pp. 424-433, March 2013.

[7] X. M. Zhang, E. B. Wang, J. J. Xia, and D. K. Sung, “An estimated distance based routing protocol for mobile ad hoc networks," IEEE Transactions on Vehicular Technology, vol. 60, no. 7, pp. 3473-3484, September 2011.

[8] D. G. Reina, S. L. Toral, P. Johnson, and F. Barreno, "Hybrid flooding scheme for MANETs," IEEE Communications Letters, vol. 17, no. 3, pp. 592-595, March 2013.

[9] Y. Mylonas, M. Lestas, A. Pitsillides, P. Ioannou, and V. Papadopoulou. "Speed adaptive probabilistic flooding for vehicular ad hoc networks," IEEE Transactions on Vehicular Technology, vol. 64, no. 5, pp. 1973-1990, May 2015.

[10] A. Mostafa, A. M Vegni, and D. P. Agrawal, "A probabilistic routing by using multi-hop retransmission forecast with packet collision-aware constraints in vehicular networks," Ad Hoc Networks, vol. 14, pp. 118-129, 2014.

[11] P. Shete and R. N. Awale, "Channel quality based adaptive gossip flooding mechanism for AODV," Proc. 13th International Symposium on Modeling and Optimization in Mobile, Ad Hoc, and Wireless Networks (WiOpt), May 2015, pp. 553-559.

[12] D. G. Reina, S. L. Toral, P. Johnson, and F. Barrero, "A survey on probabilistic broadcast schemes for wireless ad hoc networks,” Ad Hoc Networks, vol. 25, pp. 263-292, 2015.

[13] R. Draves, J. Padhye, and B. Zill, "Routing in multi-radio, multi-hop wireless mesh networks," Proc. 3rd International Conference MobiCom 2004, Octorber 2004, pp. 114-128.

[14] A. P. Subramanian, M. Buddhikot, and S. Miller, "Interference aware routing in multi-radio wireless mesh networks," Proc. 2nd IEEE Workshop on Wireless Mesh Networks (WiMesh’06), September 2006, pp. 55-63.

[15] V. C. M. Borges, D. Periera, M. Curado, and E. Monteiro, "Routing metric for interference and channel diversity in multi-radio wireless mesh networks," Proc. 8th International Conference ADHOC-NOW 2009, September 2009, pp. 55-68.

[16] J. Lu, X. Wang, and L. Zhang, "Signal power random fading interference-aware routing for wireless sensor networks," Wireless Networks, vol. 20, pp. 1715-1727, Octorber 2014.

[17] O. Dousse, P. Thiran, and M. Hasler. "Connectivity in ad-hoc and hybrid networks," Proc. Twenty-First Annual Joint Conference of the IEEE Computer and Communications Societies, vol. 2, IEEE, 2002, pp. 1079-1088.

[18] J. Sarker and R. Jantti, "Connectivity modeling of wireless multihop networks with correlated and independent factors," Proc. 6th International Conference on Advanced Communication Technology, vol. 1, 2004, pp. 474-479.

[19] T. S. Rappaport, Wireless Communication: Principles and Practice, Prentice Hall, 1999.

[20] A. Goldsmith, Wireless Communications, Cambridge University Press, 2005.

[21] S. Shakkottai, T. S. Rappaport, and P. C. Karlsson, "Cross-layer design for wireless networks," IEEE Communications Magazine, pp. 74-80, Octorber 2003.

[22] G. Bianchi, L. Frantta, and M. Oliveri, "Wireless LAN Medium Access Control (MAC) and Physical Layer (PHY) specifications," Proc. IEEE Symp. 7th Personal, Indoor and Mobile Radio Coomunications, IEEE Press, October 1996.

[23] P. J. Shete, R. N. Awale, and S. Y. Ket, "Channel quality aware cross-layer design based rate adaptive MAC for improving the throughput capacity of multi-hop ad hoc networks," Ad Hoc Networks, vol. 63, pp. 45-61, 2017.

[24] G. Grimmett, "What is Percolation?" in Percolation. Grundlehren der mathematischen Wissenschaften (A Series of Comprehensive Studies in Mathematics), vol. 321. Springer, Berlin, Heidelberg, 1999.

[25] S. Ross, Introduction to Probability Models, Elsevier, 2004.

[26] S. J. Russell and P. Norvig, Artificial intelligence: a modern approach (International Edition), Pearson, 2002.

[27] C. Bettstetter, "On the connectivity of ad hoc networks," The computer journal, vol. 47, no. 4, pp. 432-447, 2004.

[28] M. Singh and S. Soni, "Spatial correlation-based clustering in wireless sensor network," International Journal of Engineering and Technology Innovation, vol. 8, no. 4, pp. 294-306, 2018.

[29] M. Rajgor, P. Shete, and R. N. Awale, "Probabilistic energy efficient routing protocol for wireless sensor network," Proc. International Conference on Communication, Information \& Computing Technology (ICCICT), February 2018, pp. 1-6.

Copyright $\odot$ by the authors. Licensee TAETI, Taiwan. This article is an open access article distributed under the terms and conditions of the Creative Commons Attribution (CC BY-NC) license (https://creativecommons.org/licenses/by-nc/4.0/). 